Cinémas

Revue d'études cinématographiques

Journal of Film Studies

\title{
Le montage : de la vision à l'action
}

\section{Jean-Philippe Uzel}

Volume 9, numéro 1, automne 1998

Les Dispositifs de médiation au cinéma

URI : https://id.erudit.org/iderudit/024773ar

DOI : https://doi.org/10.7202/024773ar

Aller au sommaire du numéro

Éditeur(s)

Cinémas

ISSN

1181-6945 (imprimé)

1705-6500 (numérique)

Découvrir la revue

Citer cet article

Uzel, J.-P. (1998). Le montage : de la vision à l'action. Cinémas, 9(1), 63-78.

https://doi.org/10.7202/024773ar

\section{Résumé de l'article}

Cet article a pour ambition de montrer en quoi le montage, que l'on décrit souvent comme l'essence du langage cinématographique, est un dispositif de médiation de la vision et de l'action. Walter Benjamin, en élargissant les analyses de Eisenstein sur le « montage des attractions ", fut un des premiers théoriciens à souligner que le choc visuel du montage obligeait le spectateur à réagir sur le plan politique et à adopter un comportement progressiste. 


\title{
Le montage: de la vision à l'action
}

\author{
Jean-Philippe Uzel
}

\begin{abstract}
RÉSUMÉ
Cet article a pour ambition de montrer en quoi le montage, que l'on décrit souvent comme l'essence du langage cinématographique, est un dispositif de médiation de la vision et de l'action. Walter Benjamin, en élargissant les analyses de Eisenstein sur le "montage des attractions", fut un des premiers théoriciens à souligner que le choc visuel du montage obligeait le spectateur à réagir sur le plan politique et à adopter un comportement progressiste.
\end{abstract}

\section{ABSTRACT}

The aim of this article is to show how montage, often described as the essence of cinematographic language, constitutes an apparatus for the mediation of vision and action. Walter Benjamin, building on Eisenstein's analysis of the "montage of attractions," was one of the first theorists to stress that the visual shock of montage obliges the spectator to react politically and to adopt a progressive mode of behaviour.

Jonathan Crary, dans son magistral essai Techniques of the Observer, a montré comment l'apparition du cinématographe, à la fin $\mathrm{du}$ XIX ${ }^{\mathrm{e}}$ siècle, venait parachever la mutation de la vision qui s'était opérée dans les années 1810-1830 à la faveur des transformations sociales, culturelles et techniques qui marquèrent le début de la modernité. À la vision objective de la camera obscura, telle qu'elle s'était constituée à la Renaissance, les premières décennies du XIX siècle vont substituer une vision subjective qui ne prend plus comme objet de référence le monde, mais les conditions physiologiques de la vision elle-même. Ce 
changement de paradigme se manifeste tout d'abord dans la philosophie de la perception des romantiques (Goethe, Schopenhauer...), qui remettent en cause la neutralité contemplative de l'observateur cartésien au profit d'un spectateur producteur de sa propre vision. Désormais, l'œeil n'est plus le miroir de la nature, mais le lieu d'où procèdent la lumière et les couleurs. Parallèlement à ces réflexions spéculatives ont lieu les premières expériences scientifiques (de Johannes von Müller, Charles Bell, François Magendie...) qui permettent à l'optique physiologique de prendre forme. Mais ce sont certainement les nouveaux dispositifs techniques de vision qui, selon Crary, matérialisent le plus franchement cette transformation de l'observateur. De façon plus radicale que la photographie, qui aura du mal à se détacher de l'illusion réaliste de la camera obscura, le stéréoscope que Wheatstone et Brewster mettent au point dans les années 1820 va, pour la première fois, libérer la vision de tout référent extérieur (Crary, p. 116-136). C'est ce nouveau dispositif technique (suivi de beaucoup d'autres jusqu'à l'invention du cinéma : phénakistiscope, kaléidoscope, zootrope...) qui façonnera le spectateur moderne et qui orientera l'évolution des arts visuels, de Turner aux impressionnistes.

Jonathan Crary, reprenant les analyses de Michel Foucault sur le panoptique de Bentham, insiste sur le fait que les nouveaux dispositifs de vision s'apparentent également à des dispositifs d'action qui débouchent sur une nouvelle forme de contrôle social. Le regard du spectateur, tout en se libérant de l'illusion objectiviste, se trouve confronté à une surenchère de stimuli qui le manipulent et l'asservissent. L'évolution de la vision est en effet concomitante des débuts de l'âge industriel et du processus de normalisation des individus. C'est au moment où le spectateur atteint son autonomie que le sujet politique se trouve menacé dans sa liberté. Tel est le grand paradoxe de la modernité que Walter Benjamin a si bien identifié dans ses travaux sur le Paris du XIX siècle et que Crary, même s'il reconnaît à l'encontre Foucault la coïncidence entre la "société de surveillance" et la "société du spectacle» (p. 18), tend à dissiper quelque peu. Ce paradoxe s'incarne, selon Benjamin, dans le personnage baudelairien du flâneur, à la fois observé et observateur, mais aussi, 
et surtout, dans le spectateur de cinéma, qui parvient à se distraire tout en subissant un matraquage idéologique incessant. A partir des années vingt, en effet, le problème de l'observateur ne se pose plus dans les mêmes conditions qu'au siècle précédent. Le cinéma et la radio se sont imposés comme des médias de masse et jouent un rôle de premier plan dans les grands événements politiques de l'époque: la Première Guerre mondiale, la révolution d'Octobre, la montée des fascismes. La "fantasmagorie" (Benjamin, 1971a, p. 129) des passages parisiens et des Expositions universelles font soudain place au choc des actualités filmées.

Devant la manipulation des masses par les régimes totalitaires, Benjamin va quitter le champ de l'analyse historique pour celui de l'engagement politique. Dès les années trente, il prend position à plusieurs reprises sur la question des rapports entre l'art et la politique qui, selon ses propres dires, n'a guère évolué depuis Platon. Deux interventions se distinguent des autres par leur importance: tout d'abord sa conférence, "L'auteur comme producteur » (1978), prononcée en 1934 à l'Institut pour l'étude du fascisme à Paris, puis son célèbre essai de 1936, "L'œuvre d'art à l'ère de sa reproductibilité technique " (1971a), dont l'épilogue appelle à une politisation de l'art visant à contrer l'esthétisation de la politique et de la guerre à laquelle se livre le fascisme. Benjamin, dès lors, va chercher à mettre le cinéma au service de la conscience sociale des masses - car jusqu'alors, comme il le reconnaît lui-même, seuls la vedette et le dictateur sortent vainqueur devant la caméra (1971a, p. 192). Mais une question se pose aussitôt à l'esthète qu'est Benjamin : comment ne pas sacrifier la qualité esthétique d'une œuvre à sa tendance politique? Une cause, aussi fondée soit-elle, ne justifie jamais, aux yeux de Benjamin, d'instrumentaliser la création. L'orientation politique d'une œuvre n'est pas, insiste-t-il, dissociable de sa qualité esthétique; aussi va-t-il s'efforcer «[...] de donner une forme véritablement actuelle aux problèmes de la théorie esthétique: et cela de l'intérieur, en évitant toute relation "non médiatisée» à la politique " (1991, p. 124) comme il le confie à Horkheimer alors qu'il achève la rédaction de "L'œuvre d'art à l'ère de sa reproductibilité technique». 


\section{Le montage comme médiation art-politique}

Pour repenser le lien entre l'art et la politique à l'aune des nouveaux médias de diffusion, Benjamin entend se détacher des méthodes traditionnelles d'analyse de l'image, en termes de contenu et de forme, que lui offre l'histoire de l'art. Il suffit, pour s'assurer de la nouveauté de ses analyses, de comparer son essai à celui que l'historien de l'art Erwin Panofsky publie la même année sous le titre "Style et matière du septième art» (1996). Dans ce dernier, Panofsky reconnaît le rôle social que joue le cinéma: "Que cela nous plaise ou pas, il n'existe rien qui façonne plus que les films l'opinion, le goût, la langue, l'habillement, le comportement et jusqu'à l'apparence physique d'un public qui se monte à plus de 60 pour 100 de la population mondiale" (p. 110-111). Mais au moment d'expliquer comment le cinéma parvient à influencer les comportements sociaux, il ressort son outillage traditionnel d'historien de l'art et analyse l'image cinématographique en termes iconographiques et symboliques. Il n'hésite pas à déclarer que le sens du cinéma découle d'une "iconographie immuable" (p. 126) qui s'incarne à travers certaines figures, celle de la vamp, de la jeune fille pure, du méchant, etc., qui influencent, par effet mimétique, les comportements du public. Il est bien évident que c'est ce genre de raccourci entre l'art et la société que Benjamin veut éviter. Pourtant, malgré les précautions qu'il prend, "L'œuvre d'art à l'heure de sa reproductibilité technique" fait l'objet, dès sa parution, de critiques sévères. Adorno, le chef de file de l'école de Francfort, écrit à Benjamin qu'en voulant donner à l'œuvre d'art une valeur d'usage immédiate, son texte "frise l'anarchisme" et n'est qu' "un résidu très sublimé de certains motifs brechtiens" (cité dans Benjamin, 1991, p. 134-136').

Pourtant Benjamin, loin de prôner une valeur d'usage immédiate du cinéma comme le fera Brecht, comprend que la dimension politique de ce nouveau médium passe par la technique. Il déclare, dès 1927, que "[...] les progrès importants, élémentaires de l'art ne sont ni de nouveau contenu ni de nouvelle forme - la révolution de la technique précède l'un et l'autre" (1971b, p. 16). Mais cette technique, et c'est là toute l'originalité de la position benjamienne, n'est pas celle du cinématographe des 
frères Lumière, qui enregistre et projette des images en mouvement. C'est avant tout la technique du montage, autrement dit dans ce qui fait la spécificité du langage filmique depuis Birth of a Nation (1915) de Griffith. Le montage, selon Benjamin, est une technique nomade qui n'appartient pas en propre au cinéma et que l'on retrouve dans les arts visuels, dans la littérature et plus largement dans la culture qui émerge en ce début du $\mathrm{XX}^{e}$ siècle ${ }^{2}$. En fait, le montage est étroitement lié à ce que l'on pourrait appeler "le paradoxe du spectateur moderne" (à la fois libre et soumis) qui apparait, comme l'a montré Crary, dans les premières décennies du XIXe siècle. Mais, entre le flâneur du $\mathrm{XIX}^{c}$ siècle et le spectateur de cinéma des années vingt, un gouffre infranchissable s'est creusé, celui du déclin de la civilisation des Lumières vers la barbarie. Le choc auquel est confronté le flâneur constitue une expérience qui garde un caractère largement contemplatif et qui stimule l'imagination: "[...] aux chocs, d'où qu'ils vinssent, écrit Benjamin, Baudelaire a su opposer la parade de son être spirituel et physique" (1971a, p. 235); le choc que subit l'individu des années 1910-1920 n'est plus seulement une expérience esthétique, c'est avant tout un traumatisme qui le menace dans son intégrité physique. C'est précisément ce choc, à la fois culturel, social, psychologique et physique, que le montage cinématographique parvient à restituer : " $[\ldots]$ le cinéma correspond à des modifications profondes de l'appareil perceptif, celles mêmes que vivent aujourd'hui, à l'échelle de la vie privée, le premier passant venu dans une rue de grande ville, à l'échelle de l'histoire, n'importe quel citoyen d'un État contemporain " (1971a, p. 204, note 2).

Pour théoriser cette esthétique du choc, Benjamin va faire appel à la théorie du montage d'Eisenstein, du moins dans sa première version ${ }^{3}$, celle du «montage des attractions ». Plus d'un point commun lie les deux théoriciens et peut-être, en premier lieu, la réception difficile dont ont fait l'objet leurs écrits ${ }^{4}$. Rappelons tout d'abord qu'Eisenstein, ingénieur de formation, a toujours insisté sur la technicité du montage: "Un mot est passé des techniques dans le langage courant. Il sert à désigner l'assemblage des pièces de machines et des éléments de tuyauterie. Un si joli mot: "le montage"!» (1958, p. 16). Ce qui est encore 


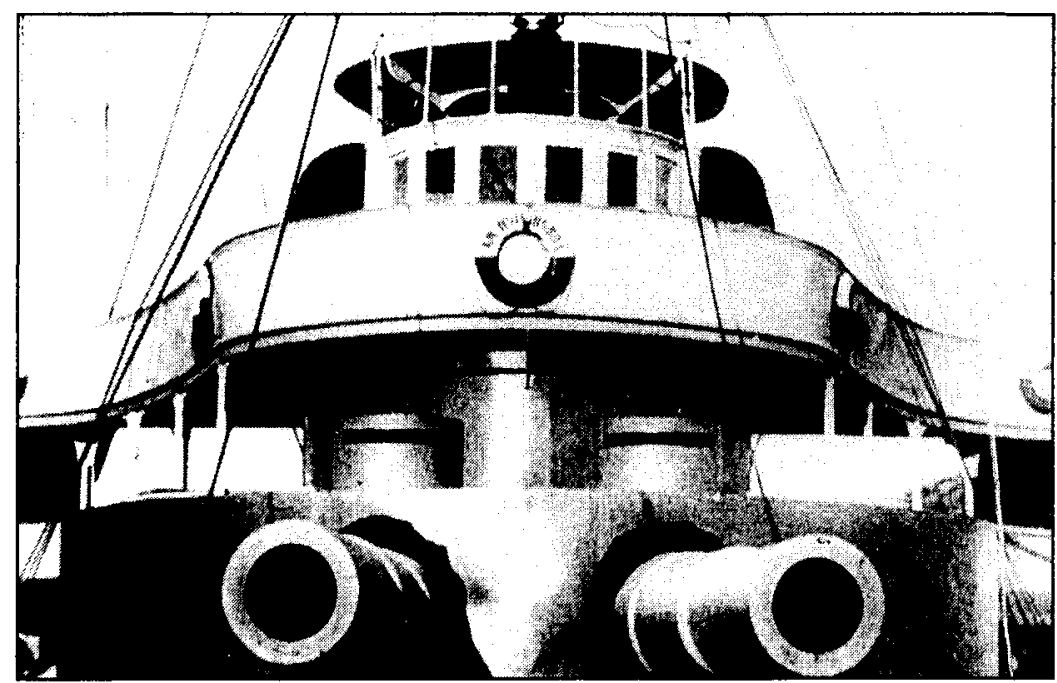

\section{Le Cuirassé Pofemkine de Serge Eisenstein (1925) \\ Collection Cinémathèque québécoise}

plus remarquable, c'est le fait qu'Eisenstein, anticipant l'analyse de Benjamin, place le montage des attractions au cour de la modernité en rappelant que le premier mot de l'expression provient du music-hall et le second, de l'industrie et que «[...] tous deux, au reste, plongent leurs racines dans l'urbanisme" (1958, p. 19), c'est-à-dire dans cette nouvelle culture des mégalopoles (Berlin, New York, Paris, etc.) conçues à l'échelle des masses.

Benjamin insiste également sur le fait que l'effet choc du montage n'est pas apparu pour la première fois dans le cinéma, mais dans les arts visuels et la littérature: "le dadaïsme cherchait à produire, par les moyens de la peinture (ou de la littérature), les mêmes effets que le public demande maintenant au cinéma " $\left(1971 \mathrm{a}\right.$, p. $203^{5}$ ). Là encore, Eisenstein fait figure de précurseur. Dans son célèbre article, "Le montage des attractions", publié en 1923 dans la revue Lef, il insiste sur le fait que le montage des attractions est né dans le théâtre; tout d'abord dans le théâtre du Grand-Guignol puis, dans un sens plus politique, dans l' "agit-théâtre» du Proletkult. Plus tard, il reconnaîtra qu'il faut remonter encore plus loin et chercher les racines de cette 
technique dans le music-hall et même dans le cirque et les spectacles forains. Point de vue que confirme Jacques Aumont dans son ouvrage sur le montage chez Eisenstein :

[...] l'attraction, originellement, c'est l'attraction du music-hall, un moment fort de spectacle, relativement autonomisable, faisant appel à des techniques de représentation qui ne sont pas celles de l'illusion dramatique, à des formes de spectacle plus agressives (cirque, music-hall, baraque foraine...) (p. 57).

Mais le metteur en scène admet également que le choc du montage est utilisé dans les œuvres provocatrices des artistes dadaïstes et constructivistes. Selon lui, le montage des attractions de l'agit-théâtre entretient " [...] une analogie parfaite avec le "potentiel figuratif" de Grosz ou les éléments de photo-illustration de Rodtchenko" (Eisenstein, 1974, p. $118^{\circ}$ ).

C'est cette explication que Benjamin va reprendre à son compte pour l'approfondir - même si, étrangement, il ne fait aucune référence aux photomontages constructivistes (Rodtchenko) ou dadaïstes (Heartfield, Hausmann, Höch....). Il explique la force subversive des ouvres dadaïstes par le fait que les artistes parviennent, en introduisant dans la représentation picturale des éléments hétérogènes (des billets, des bouts de cigarettes, des bobines...), à briser l'aura de l'œuvre et à empêcher tout recueillement contemplatif chez le spectateur: "Leurs poèmes sont des "salades de mots", ils contiennent des obscénités et tout ce qu'on peut imaginer comme détritus verbaux. De même leurs tableaux, sur lesquels ils collaient des boutons et des tickets"(1971a, p. 203). Benjamin donne l'exemple des tableaux-collages de Kurt Schwitters dans lesquels " [...] la plus minuscule parcelle authentique de la vie quotidienne dit plus de chose que la peinture» (1978, p. 118). Ainsi, en déjouant les habitudes perceptives du spectateur, le montage oblige ce dernier à réagir: "[...] pour une bourgeoisie dégénérée, la rentrée en soi-même était devenue une école de comportement asocial, avec le dadaïsme, la diversion devient un exercice de comportement social" (1971a, p. 203). La distraction (au sens de diversion plus que de divertissement) créée par le choc du montage débouche sur la praxis politique. 


\section{L'effet choc du montage}

Le passage de la désacralisation de l'art à l'engagement politique peut, pour le moins, sembler aléatoire. Pour en admettre l'efficace, il faut comprendre que le choc du montage cinématographique ne fait que "reproduire" le choc social et culturel de l'époque - qui, nous l'avons déjà dit, n’a plus grand-chose à voir avec celui que pouvait éprouver le flâneur baudelairien. Ce choc, c'est d'abord celui de la Première Guerre mondiale qui va marquer, dans sa chair, toute une génération. Rappelons-nous que les artistes qui se réunissent en 1916 au cabaret Voltaire de Zurich, et qui vont donner naissance au mouvement dada, sont d'abord des "traumatisés" des tranchées. Brigid Doherty, dans une étude récente, affirme qu'il faut prendre au sérieux le vers de Raoul Haussman dans lequel il déclare: «Voyez: nous sommes tous neurasthéniques!» Elle soutient que le choc traumatique que produit le monrage dada n'est que le prolongement du traumatisme physique et psychique du spectateur ${ }^{7}$ :

The "real situation " that dada will force its viewers to recognize is emphatically a "material " one, a bodily one. In dada's synthetic cinema of painting, in montage, marvelous objects made of mundane "real material" "correspond organically" to the disposition of the viewer's own body, a body so stiff it threatens to shatter. Dada, says Hausmann, compels the viewer's bodily identification with the traumatic shocks it simulates (p. 131-132).

Dans son article "Sur quelques thèmes baudelairiens" (1971a), Benjamin s'appuyait sur l'étude de Freud, Au-delà du principe de plaisir (1920), pour donner une assise psychologique à sa théorie du choc visuel du flâneur parisien. Brigid Doherty suggère que Sándor Ferenczi, le collègue de Freud, qui avait traité des névrosés dans les hôpitaux militaires de la Première Guerre mondiale, nous offre des concepts beaucoup plus opératoires pour comprendre l'esthétique traumatique de dada (p. 89). Elle nous rappelle que Ferenczi affirmait que les symptômes névrotiques de certains de ses patients ne pouvaient être représentés fidèlement que par le cinéma: "[...] the gait of the trembler is most remarkable; he gives the impression of spastic 
paresis; but the varying mixture of tremor, rigidity, and weakness occasions quite peculiar gaits, possibly only to be reproduced cinematographically» (Ferenczi, cité par Doherty, p. 131, note 63). Mais Benjamin avait également saisi la profonde mutation que l'expérience esthétique avait subie dans les premières décennies du siècle, aussi bien au niveau des arts visuels que du cinéma:

\begin{abstract}
De spectacle attrayant pour l'œil ou de sonorité séduisante pour l'oreille, l'œuvre d'art avec le dadaïsme se fit choc. Elle heurta le spectateur ou l'auditeur. Elle acquit un pouvoir traumatisant. Et elle favorisa de la sorte le goût du cinéma, qui possède, lui aussi, un caractère de diversion en raison des chocs provoqués chez le spectateur par les changements de lieux et de décors (1971a, p. 204 ; c'est nous qui soulignons).
\end{abstract}

Ce trauma, ce n'est plus, pour Benjamin, celui du flâneur, mais bien celui du poilu.

Cette conception de l'esthétique choc du montage est déjà tout entière chez Eisenstein, qui en fait la clé de voûte de l'efficacité politique du théâtre et du cinéma. Selon lui, le montage des attractions a pour objectif «[...] le façonnage [du] spectateur dans le sens désiré à travers toute une série de pressions calculées sur son psychisme" (1974, p. 127). Il s'agit littéralement selon lui "[...] de saisir par les cheveux le spectateur abasourdi et, d'un geste impérieux, de le mettre face aux problèmes actuels" (1974, p. 52). Eisenstein écrira en 1945 que s'il avait mieux connu la théorie de Pavlov dans les années vingt, il aurait donné au montage des attractions le nom de "théorie des existants esthétiques" (1958, p. 19). Cette théorie du choc ressort déjà très bien dans la première définition du montage des attractions de 1923:

Est attraction (du point de vue du théâtre) tout moment agressif du théâtre, c'est-à-dire tout élément de celui-ci soumettant le spectateur à une action sensorielle ou psychologique vérifiée au moyen de l'expérience et calculée mathématiquement pour produire chez le spectateur certains chocs émotionnels qui, à leur tour, une fois réunis, conditionnent seuls la 
possibilité de percevoir l'aspect idéologique du spectacle montré, sa conclusion idéologique finale (1974, p. 117).

Nous percevons ici très nettement la dimension expérimentale (au sens scientifique du terme) qu'Eisenstein souhaite donner à son travail. Il admet d'ailleurs, en bon ingénieur, que le calcul des réflexes du public peut faire l'objet d'erreur. La fameuse scène qui clôt La Grève (1924) et que l'on donne toujours comme l'exemple caractéristique du montage des attractions n'a pas eu, selon lui, l'effet souhaité, le profil psychologique du public ayant fait l'objet d'une mauvaise évaluation:

La scène de l'abattoir n'a pas eu cet effet sanglant sur le public ouvrier pour la simple raison que dans l'esprit de l'ouvrier le sang de bœuf est associé au premier chef à l'usine de récupération du sang des abattoirs! Quant à l'effet sur le paysan habitué à égorger lui-même le bétail, il a été parfaitement nul (1974, p. 26).

Si la démarche expérimentale qu'adopte Eisenstein se retrouve chez les autres cinéastes soviétiques, et découle en fait du matérialisme historique, sa conception du montage reste tout à fait originale. Le montage des attractions, par exemple, n'est en aucun cas comparable à "la géographie créatrice» que Koulechov cherche à créer, c'est-à-dire "[...] un nouvel espace, un espace filmique sans existence dans la réalité" (Koulechov, cité dans Chateau, p. 33). Cette conception de la réalité filmique reste encore très proche de la vision subjective du XIX ${ }^{e}$ siècle. L' «effet Koulechov" s'inscrit tout à fait dans la refonte de la vision décrite par Jonathan Crary puisque le spectateur est censé produire un sens qui n'existe pas dans les plans eux-mêmes, mais seulement dans son esprit. De même, le «Ciné-œil» de Vertov est très éloigné du cinéma eisensteinien. Loin de vouloir choquer le spectateur, il s'agit au contraire de lui dévoiler le processus de fabrication du film. Pour Vertov, le montage a une dimension éthique qui consiste non pas à cacher ses moyens (contrairement aux attractions dont le ressort essentiel est la surprise), mais à les exposer en en faisant le véritable objet du film: "Si, dans L'Homme à la caméra, ce n'est pas le but qui est mis en 
relief, mais le moyen, c'est de toute évidence parce que le film avait, entre autres, la tâche de présenter ces moyens au lieu de les dissimuler comme il était d'usage dans les autres films" (p. 208). Dans L'Homme à la caméra (1929), tout le film se fait par la médiation de la caméra de l'opérateur Mikhaill Kaufman. Cette articulation entre le voir et le savoir nous renvoie aux premiers temps du cinéma lorsque le regard du spectateur était assisté par certains dispositifs de vision (le trou de serrure, le microscope...). Ce n'est d'ailleurs pas un hasard si le montage apparaît en même temps que ces dispositifs de vision dans les films La Loupe de Grand'Mère (1900) et Ce quion voit dans un télescope (1900) du réalisateur britannique G. A. Smith (Sadoul, 1963 , p. 42-43).

\section{Au-delà de la vision subjective}

Le montage des attractions, cette "[...] série de coups portés à la conscience et aux sentiments du spectateur " (Eisenstein, 1974, p. 127), crée quant à lui une discontinuité qui suspend le procès de sens et vient briser la trame narrative et expressive du film. Eisenstein condamnera sévèrement le Kinoglaz qu'il jugera encore trop axé sur la contemplation: "Ce n’est pas un "Cinéceil " qu'il nous faut, mais un "Ciné-poing" [...] nous ne devons pas contempler mais agir» (1974, p. 153). Il demande expressément à ces lecteurs de ne pas confondre «[...] le montage des attractions et son procédé de confrontation, avec l'habituel parallélisme du montage dans l'exposition du sujet" (p. 133). Benjamin arrive aux mêmes conclusions lorsqu'il compare la peinture (de la Renaissance jusqu'à la fin du XIX siècle) et le cinéma. Selon lui, l'attitude esthétique contemplative, héritée du romantisme, est désormais caduque. Alors que la peinture était tout entière harmonie et conciliation, le cinéma fonctionne sur le mode traumatique de la distraction: " [...] la peinture, nous dit Benjamin, invite à la contemplation; en sa présence on s'abandonne à ses associations d'idées. Rien de tel au cinéma [où] la succession des images interdit toute association dans l'esprit du spectateur» (1971a, p. 204). Leffet de choc du montage vient heurter les habitudes contemplatives du public. Celui-ci ne peut plus se projeter dans un espace fictionnel, mais est sans 
cesse renvoyé au comportement névrotique qui le caractérise. Alors que le mode de contemplation passif de la peinture était devenu synonyme d'une position politiquement conservatrice, le montage cinématographique appelle le spectateur à réagir sur le plan social, l'oblige à adopter un comportement progressiste. C'est également cet effet que l'on retrouve dans le dadaïsme qui ne se contente pas de remettre en cause les conventions picturales, comme avait pu le faire l'impressionnisme au XIX ${ }^{\mathrm{e}}$ siècle, mais subvertit l'institution des Beaux-Arts et la conduite esthétique désintéressée qu'elle supposait. La différence est si radicale entre l'impressionnisme, d'un côté, et le cinéma et le dadaïsme, de l'autre, que Benjamin se demande si on est encore en droit de parler d' "art», pour qualifier ces derniers: "[...] la fonction de l'art se trouve bouleversée. Au lieu de reposer sur le rituel ${ }^{8}$, elle se fonde désormais sur une autre forme de praxis: la politique" (1971a, p. 181).

C'est en effet dans les deux cas l'efficacité politique qui prime - même si l'éducation politique que prône Eisenstein est étrangère au dadaïsme, qui est avant tout un nihilisme. On retrouve chez ces deux théoriciens, la volonté de façonner un "nouveau spectateur ", qui puisse réagir immédiatement au contexte sociopolitique de la société de masse. Eisenstein et Benjamin sont parmi les premiers à comprendre que le spectateur romantique et avec lui la culture visuelle moderne se sont évanouis à l'aube $\mathrm{du} \mathrm{XX}^{\mathrm{e}}$ siècle. Cette transformation, ils l'acceptent sans aucune nostalgie et en reconnaissent même les aspects positifs : "La masse est la matrice d'où sort à l'heure actuelle tout un ensemble d'attitudes nouvelles à l'égard de l'œuvre d'art. La quantité est devenue qualité" (Benjamin, 1971a, p. 205). Ils passent aux profits et pertes l'autonomie de l'observateur moderne: la vision n'est plus un objet de connaissance, elle est avant tout un moyen d'action. Qu'il s'agisse des références à la réflexologie chez Eisenstein ou à la psychanalyse chez Benjamin, la construction du sens est abandonnée au profit du réflexe et du trauma, des chocs émotionnels et nerveux du spectateur.

Une telle posture intellectuelle s'accompagne d'une série de paradoxes, au premier rang desquels cette dialectique incessante entre la théorie et la pratique, entre le descriptif et le prescriptif. 


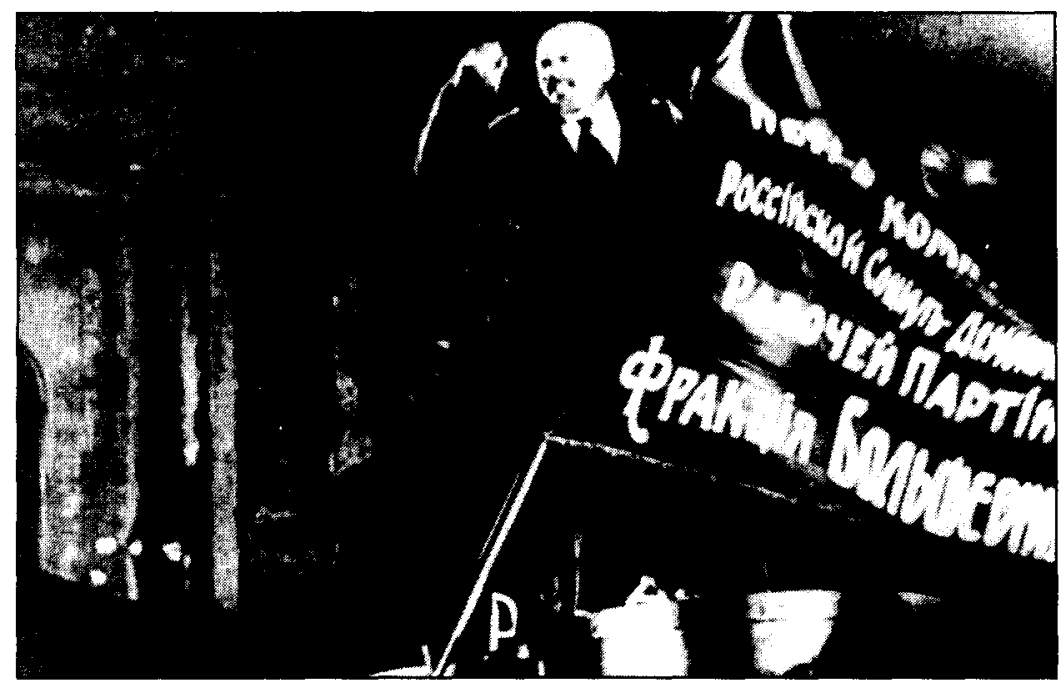

\section{Ocfolbre de Serge Eisenstein (1928)}

Collection Cinémathèque québécoise

Eisenstein a plus écrit sur le montage des attractions qu'il ne l'a mis en pratique dans ses films; Benjamin a entrepris la rédaction d'un de ses principaux écrits théoriques pour des raisons politiques. Ce paradoxe central se répercute, tout particulièrement chez Benjamin, sur la question de la médiation. Nous pourrions le formuler ainsi: la volonté de passer de la vision à l'action sans la médiation du savoir (l'urgence des événements l'interdit), l'oblige, sur le plan théorique, à multiplier les médiations. C'est ce paradoxe, croyons-nous, qui explique que ses écrits seront rejetés à la fois par les penseurs marxistes, totalement étrangers à la question de la médiation, comme Adorno", et par la récente "sociologie de la médiation" (Hennion et Latour, 1996) qui lui reproche son messianisme politique. Pourtant, il nous semble que Benjamin - dès lors qu'on se donne la peine de le lire et qu'on ne se contente pas des nombreux lieux communs qui entourent son texte (la perte de l'aura, l'invasion de la valeur d'échange...) - parvient, grâce au montage, à relever le défi de la médiation. Il montre que la dimension politique du cinéma ne passe pas forcément par sa forme ou son contenu et n'est pas forcément synonyme d'instrumentalisation. Mais 
au-delà de la relation de l'art et de la politique, "L'œuvre d'art à l'ère de sa reproductibilité technique» est la première réflexion, à notre connaissance, qui s'interroge sur la fin de la vision subjective ou du moins sur les risques qu'elle fait courir au spectateur du XX $X^{e}$ siècle. Benjamin comprend que la tension entre surveillance et spectacle ne trouve plus, dans les années vingt et trente, sa solution esthétique (comme c'était encore le cas chez Baudelaire) que dans le fascisme. Tel est, nous semble-t-il, le sens de ses dernières phrases, lorsqu'il affirme que l'esthétisation de la guerre que prône Marinetti n'est que «[...] la parfaite réalisation de l'art pour l'art [...]" et qu'aujourd'hui "[l'humanité] est devenue assez étrangère à elle-même pour réussir à vivre sa propre destruction comme une jouissance esthétique de premier ordre » (1971a, p. 210).

\section{Université du Québec à Montréal}

\section{NOTES}

1 Le reproche d'Adorno semble peu fondé puisque la revue Das Wort, dont Brecht était un des principaux rédacteurs, refusa de publier l'essai de Benjamin. De plus, à la lecture du Journal de Travail de Brecht, on voit bien que le sens du texte de Benjamin lui échappe totalement: "[Benjamin] part de quelque chose qu'il appelle aura, en rapport avec le rêve (le rêve diurne). Il dit: quand on sent un regard dirigé sur soi, même dans son dos, on cherche à y répondre (!). L'espoir d'être regardé par ce qu'on regarde crée l'aura. Celle-ci tendrait à dépérir depuis peu, conjointement avec le cultuel. B.[Benjamin] a fait cette découverte en analysant le cinéma, où l'aura s'évanouit à cause de la reproductibilité des ceuvres - pure mystique, malgré la posture antimystique. C'est donc ainsi qu'on adapte la conception matérialiste de l'histoire! C'est assez épouvantable" (Paris: L'Arche, 1976, p. 15).

2 À l'inverse de Benjamin, Panofsky n'accorde aucune importance au montage et n'hésite pas à écrire que "[...] tous les films tournés entre 1900 et 1910 préfigurent tous les sujets et toutes les époques du cinéma que nous connaissons depuis" (E. Panofsky, "Style et matière du septième art », Trois Essais sur le style, Paris: Le Promeneur, 1996, p. 120). Les innovations techniques qu'il note chez D.W. Griffith sont celles mises en ouvre dans ses premiers films de 1909, essentiellement le plan d'ensemble, le gros plan et le flashback.

3 Sur l'évolution de la théorie du montage chez Eisenstein, qui prendra plusieurs formes différentes (montage vertical, montage intellectuel...), on pourra se référer à l'ouvrage très complet de Jacques Aumont, Montage Eisenstein (Paris: Albatros, 1979).

4 Pierre Beylot nous rappelle que l'œuvre théorique d'Eisenstein, et tout particulièrement sa théorie du montage, a été boudée par les plus grands théoriciens du cinéma, qui n'y ont vu, à l'instar de Christian Metz, que la victoire de l'" esprit manipulateur" ("S. M. Eisenstein: le chantre du montage-roi ", CinémAction, n" 72, 1994, p. 39). 
5 Georges Sadoul, dans un article de 1963, fait la remarque suivante au sujet de l'ambiance artistique des années 1910-1920: "Créer de l'art par le montage, l'assemblage, ou le collage d'éléments enregistrés ou préexistants dans lesquels l'artiste n'était pas personnellement intervenu, cette idée "était dans l'air" depuis le début du $\mathrm{XX}^{\prime \prime}$ siècle, dans tous les milieux d'avant-garde européens. Ainsi en témoignèrent durant les années 1910-1920 les "papiers collés" de Picasso et Braque [...], les sculptures assemblant des objets manufacturés (ou autres) de Duchamp, Max Ernst, etc." ("L'actualité de Dzigga Vertov", Cabiers du cinéma, n"144, 1963b, p. 24-25).

6 La relation entre dadaïstes (Grosz) et constructivistes (Rodtchenko) a longtemps été ignorée par l'histoire de l'art moderne et commence tout juste à faire l'objet de recherches spécifiques. En ce qui concerne le cinéma, nous savons que Hans Richter, qui a réalisé l'œuvre cinématographique la plus importante du dadaïsme, avait rencontré à plusieurs reprises Eisenstein et que les deux artistes se vouaient une admiration réciproque (Richter, cité dans Philippe Sers, Sur Dada, Nîmes: Jacqueline Chambon, 1997, p. 207-210).

7 Le trauma est un violent choc émotionnel qui modifie la personnalité en la sensibilisant aux chocs de même nature. On peut donc affirmer que le "traumatisé" est aussi, peu ou prou, un "traumaphile". Le traumatisme est l'ensemble des troubles, aussi bien physiques que psychiques, qui résultent du trauma.

8 L'usage du terme "rituel " ne se limite pas, chez Benjamin, à la sphère religieuse, mais s'applique également au monde des Beaux-Arts. Il le dit très clairement: le rituel lié au beau va se substituer, à partir du XVIIr siècle, au rituel religieux qui a entouré la réception des ouvres d'art: "[si] les plus anciennes cuvres d'art naquirent au service d'un rituel, magique d'abord, puis religieux [...] cette liaison fondamentale est encore reconnaissable, comme un rituel sécularisé, à travers le culte voué à la beauté [...] de plus en plus, à l'unicité des phénomènes régnants dans l'image cultuelle, le spectateur tend à substituer l'unicité empirique de l'artiste ou de son activité empirique" (Poésie et Révolution, Paris: Denoël, 1971a, p. 179-180).

9 Adorno ne s'est en fait jamais vraiment débarrassé de la dialectique marxiste et de son corollaire, la primauté de l'infrastructure sur la superstructure. Il suffit pour s'en assurer de relire son commentaire du montage dadaïste: "Qu'est-ce qu'un montage? C'est une perturbation visant le sens des œuvres d'art, récusant leur légalité par une invasion de fragments de la réalité empirique [...]. L'art devient virtuellement un objet parmi les objets, il devient quelque chose dont nous ne savons pas ce que c'est» ("L'Art et les arts", L'Art dans la société d'aujourd'hui (collectif), Neuchâtel: Éditions de la Baconnière, 1968, p. 54-55).

\section{OUVRAGES CITÉS}

Adorno T. W. "L'Art et les arts", L'Art dans la société d'ałujourd'hui (collectif). Neuchâtel: Éditions de la Baconnière (1968), p. 39-57.

Aumont, Jacques. Montage Eisenstein. Paris: Albatros, 1979.

Benjamin, Walter. É crits français. Paris: Gallimard, 1991.

Benjamin, Walter. Eisais sur Bertolt Brecht. Paris : Maspero, 1978.

Benjamin, Walter. Poésie et Révolution. Paris: Denoël, 1971 a.

Benjamin, Walter. "Discussion sur le cinéma russe et l'art collectiviste en général ". Cahiers du cinéma, n" 226-227 (1971b), p. 16-17.

Beylot, Pierre. "S. M. Eisenstein: le chantre du montage-roi». CinémAction, n" 72 (1994), p. 39-49.

Brecht, Bertolt. Journal de travail: 1938-1955. Paris: L'Arche, 1976. 
Chateau, Dominique. "Le montage comme expérimentation (Koulechov, Poudovkine, Vertov)». CinémAction, n" 72 (1994), p. 32-38.

Crary, Jonathan. Techniques of the Observer. Cambridge: MIT Press, 1990.

Doherty, Brigid. "See: We Are All Neurasthenics!" or, The Trauma of Dada Montage ». Critical Inquiry, vol. 24, n" 1 (1997), p. 82-132.

Eisenstein, S. M. Au-delà des étoiles. Paris: Union générale d'éditions, 1974.

Eisenstein, S. M. Réflexions d'un cinéaste. Moscou: Éd. Langues étrangères, 1958.

Hennion, Antoine et Bruno Iatour. "L'art, l'aura et la distance selon Benjamin, ou comment devenir célèbre en faisant tant d'erreurs à la fois... ". Les Cahiers de médiologie, n"1 (1996), p. 235-241.

Panofsky, Erwin. "Style et matière du septième art", Trois Essais sur le style. Paris: Le Promeneur (1996), p. 109-145.

Sadoul, Georges. Histoire du cinćma mondial. Paris: Flammarion, $1963 \mathrm{a}$.

Sadoul, Georges. "Lactualité de Dziga Vertov». Cahiers du cinéma, n" 144 (1963b), p. 23-31.

Sers, Philippe. Sur Dada. Nîmes: Jacquelinc Chambon, 1997.

Vertov, Dziga. Articles, journaux, projets. Paris: Union générale d'éditions, 1972. 